\title{
BEHAVIORAL RESPONSES TO SHORT \\ PERIODS OF LOWERED GRAVITATIONAL FORCE \\ IN BLIND GOLDFISH
}

\author{
R. J. VON BAUMGARTEN, J. ATEMA, T. HUKUHARA, and M. ROCKER \\ Mental Health Research Institute, University of Michigan, Ann Arbor, Mich., U.S.A.
}

\begin{abstract}
The movements of blind goldfish flown in an aircraft through vertical flight patterns show a consistent correlation with the varying $g$ loads as recorded by a $g$ meter.

(2) Decreasing the $g$ load in all tested cases caused the fish to rapidly dive downwards after an approximate delay of $0.5-1.5 \mathrm{sec}$. The opposite reaction, a tilt and movement upwards, was observed in transitions from a lower to a higher $g$ load, though the latter reaction was not as pronounced.

(3) Because any potential influence of visual cues, swimming bladder reflexes, and changing barometric pressure was excluded by experimental precautions, we concluded that the response of the fish to varying $g$ loads were vestibular reflexes, probably resulting from displacements of the otoliths during vertical accelerations.

(4) It is concluded from data in the literature and from the present results, that the behavioral responses resulting from static or dynamic forces are counterdirected toward the otolith displacements caused by these forces. Consequently, the behavioral responses are counterdirected toward the gravitational pull on the animal, but must have the same direction as mechanical forces, which accelerate the whole organism and displace the otolith by inertia. It is concluded therefore, that the otolith reflexes have adaptive value only in a gravitational or centrifugal field, but are unsuitable to subserve any stabilizing function in the case of inertial forces during weightlessness.
\end{abstract}

\section{Introduction}

The semi-circular canals are commonly believed to be receptors for angular accelerations and the otoliths receptors for gravitational force only. From a purely physical standpoint, the latter force cannot be distinguished from linear acceleration of the body caused by linear displacements or applied centrifugal force (BREUER, 1891). It is possible, therefore, to counteract gravity by an opposingly directed centrifugal field. Under these circumstances otolith displacement depends solely on inertial forces.

Since this condition characterizes satellites orbiting in space, it is of particular interest to expand our knowledge of vestibular reflexes elicited by inertial and dynamic forces other than gravity. It is doubtful whether these reflexes can be studied on the ground with the aid of mechanical linear accelerators or centrifuges without being contaminated to some degree by the influence of the gravitational field of the earth $\left(g=9.81 \mathrm{~m} / \mathrm{sec}^{2}\right)$, the Coriolis-, crosscoupling-, and postrotatory effects (HowARD and Templeton, 1966). To gain maximum information, such studies should ideally be undertaken in orbiting satellite or space stations. However, for certain purposes it is possible to satisfactorily simulate these conditions in an airplane. Such comparatively inexpensive experiments could help to obtain valuable pilot data which would help to plan subsequent experiments in space. Weightlessness can momentarily be attained by flying an airplane through a parabolic Keplerian trajectory during which gravity 
approaches zero for short periods (HABER and HABER, 1950; WARD, 1957). GERATHEWOHL et al. (1957) achieved weightlessness for periods up to $45 \mathrm{sec}$ in jetplanes. We used only a light propeller-driven aircraft, which allowed us to obtain weightlessness for even shorter periods lasting 3 to $8 \mathrm{sec}$, which nevertheless proved to be long enough to show a consistent response of goldfish to the transition from high $g$ to low $g$ and vice versa.

Fish were used in these experiments because they live in a three-dimensional environment, and changes of vestibular input lead promptly to measurable changes of the fish's attitude and position in the tank. The rigid connection between head and trunk in fish allows the comparison of changes in the position of the whole body with changes of the vestibular input. Therefore, the whole body-movements of fish can serve as indicators for changes in vestibular input in much the same way as do the eye-movements in mammals. It will be shown in this paper, that goldfish consistently react to a decreasing $g$ load by quickly tilting their longitudinal axis downwards and diving down in the tank. HAwKINS (1960), who flew goldfish through parabolas, reports that they swam during full weightlessness in irregular body positions and seemed somewhat 'confused'.

\section{Technique}

Fifteen goldfish, fully anaesthetized with tricaine methane sulfonate (Sandoz, MS 222), were blinded in a preliminary operation by removing both eyes. Several days were allowed for recuperation, at the end of which the fish were placed one at a time in a

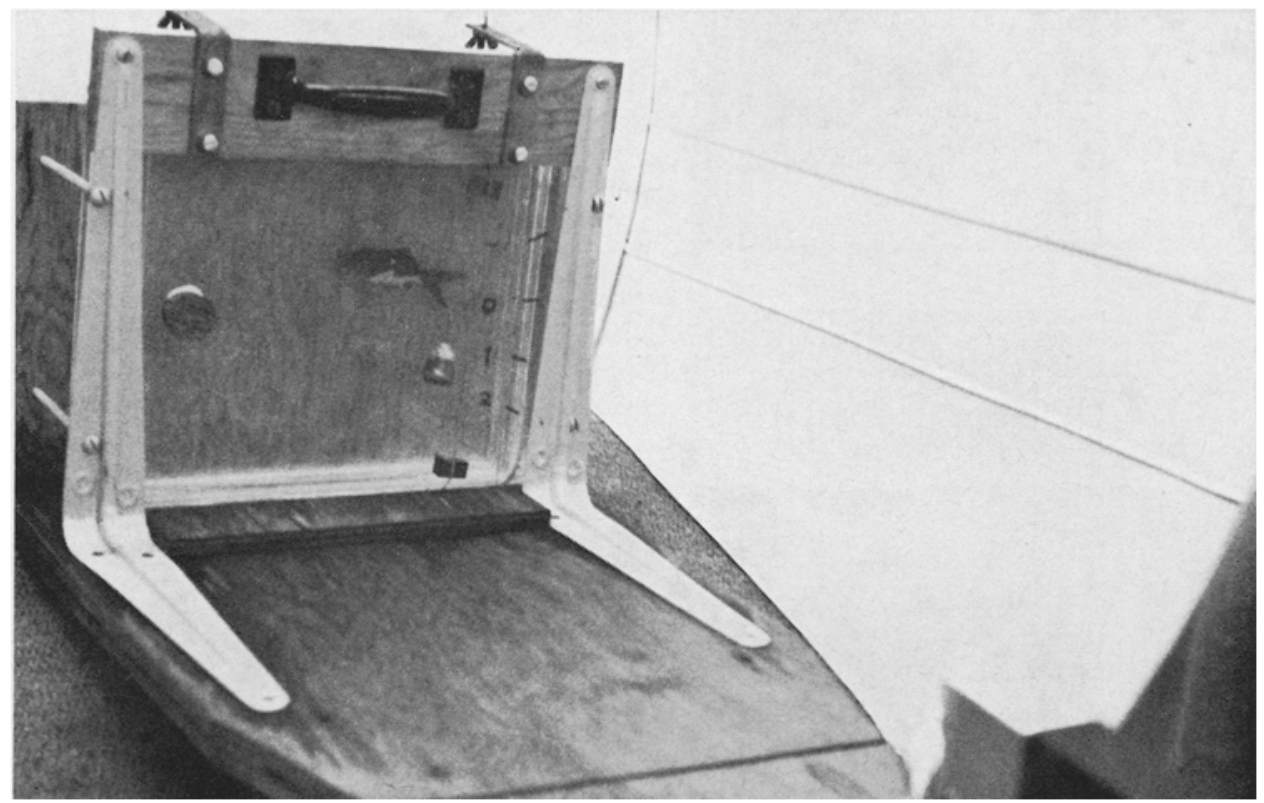

Fig. 1. Fishtank, goldfish, $g$ meter and movie camera in the luggage compartment of a Cessna 150 airplane. 
A

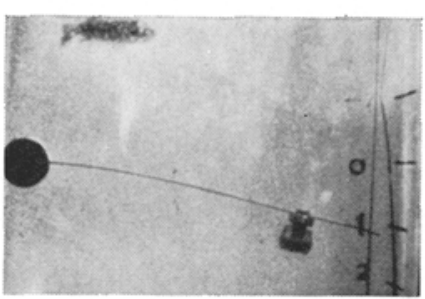

2

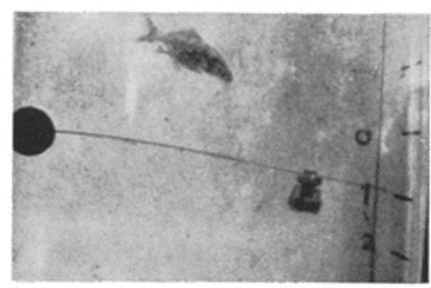

3

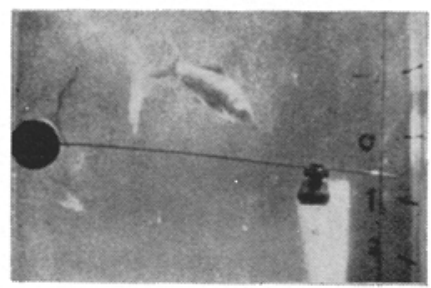

4
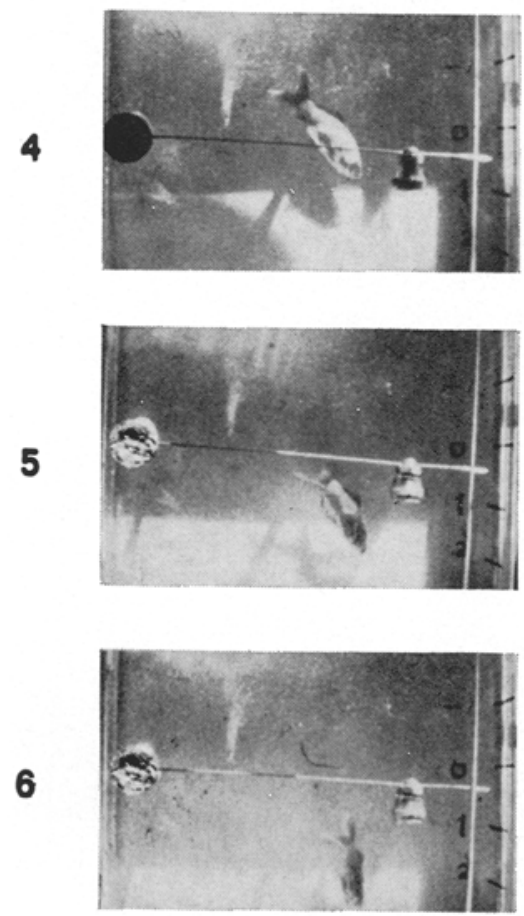

B
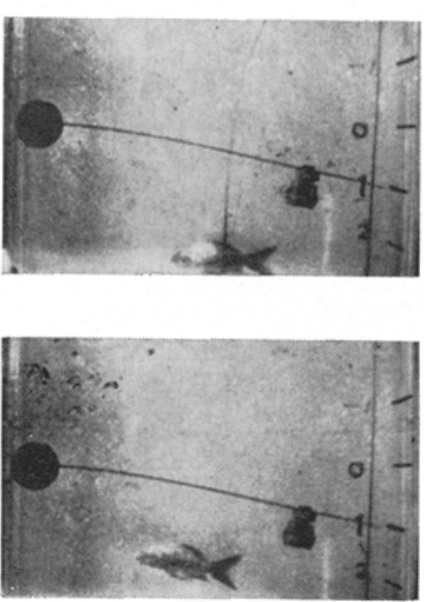

3

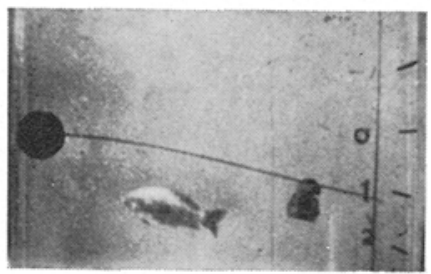

4

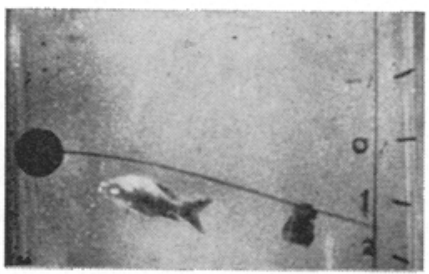

5

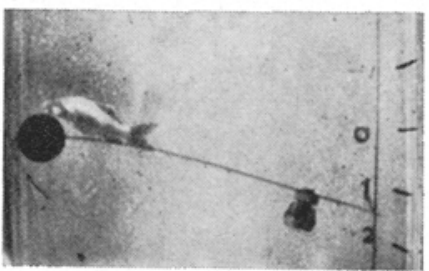

6

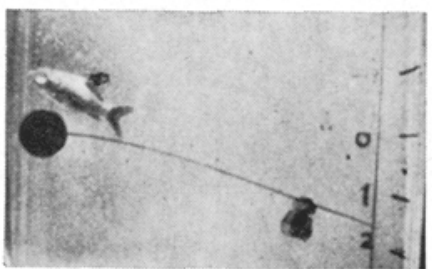


special experimental tank $25 \mathrm{~cm}$ high, $27 \mathrm{~cm}$ long, and $7 \mathrm{~cm}$ wide (Figure 1). These dimensions allowed the fish free movement in all directions except for excessive excursions in the anterior-posterior axis of the tank, the latter restriction being necessary to keep the fish within depths of field of a recording camera. To obtain a water- and airtight system, a glass plate was screwed down over the tank and the space between them sealed with silicone grease. In this way a constant pressure in the tank was maintained in the face of variations in atmospheric pressure correlated with changes in altitude. Experiments with varying amounts of enclosed air above the water level were also performed, without significant changes in test results.

A simple springtype $g$ meter was attached to the front of the tank and calibrated by marking the position of one $g$, and by inversion, 'negative one $g$ '; zero $g$ was determined by the mid point between these extremes, and smaller values by extrapolation. An $8 \mathrm{~mm}$ electrically driven movie camera with an automatic light meter was fastened to the wooden framework holding the tank. A high speed setting of 24 frames/sec produced slow motion records of fish movements and $g$ meter deflections.

Filmrecords were projected with a single frame editor-type machine, and the $g$ meter reading, tilt of the fish and its position in the tank were noted for each frame.

The fishtank and the camera were rigidly fixed in the luggage compartment of a light aircraft (Cessna 150) and the parabolic maneuvers performed at altitudes ranging between 3000 and 4000 feet above ground. To insure a smooth flight pattern, proximity to clouds and other areas of turbulence was avoided as much as possible, for even small accelerations of the plane due to external turbulence along the vertical axis were accompanied by changes in the fish's vertical orientation.

Preliminary studies determined that an entrance speed of $120 \mathrm{mph}$ and a $45^{\circ}$ initial climb, followed by a parabolic flight pattern, were suitable for the achievement of the desired period of lowered $g$ force. Higher than one $g$ loads were obtained by pulling up the airplane from a shallow dive into a $30^{\circ}$ climbing attitude. Because control records close to one $g$ were desired to facilitate evaluation of data, an effort was made to minimize the increase in $g$ load preceding and following the parabolic maneuver; this was accomplished by elongated, smooth entrances and exits. While the $g$ meter continued to be observed by both pilot and copilot and recorded by the film camera throughout the maneuver, it was possible, after some practice, to estimate zero and one $g$ loads 'by the seat of the pants'.

The flight patterns, of course, were not always identical, but the period during which a substantially lowered $g$ force was maintained varied between 3 to 5 sec. To avoid complicating our results we preferred not to lower the $g$ force to absolute

Fig. 2. Responses of a goldfish to decreasing and increasing $g$ load. The needle on the front of the fishtank always indicates the approximate $g$ force. A. 1-6 are single frames taken from an eight mm movie film at intervals of approximately $360 \mathrm{msec}$. Note the dive of the fish during decreasing $g$ load. B. 1-6 are single frames at intervals of approximately $410 \mathrm{msec}$. Note the climb of the fish during increasing $g$ load. 
weightlessness, but to keep it on the positive side. Achievement of zero $g$ often entailed the following problems: first, the water throughout the tank was disturbed by nonbuoyant air bubbles, and second, the occasional appearance of small negative $g$ forces threatened to contaminate the data. We have reason to believe that any negative $g$ force would cause blind fish to assume a belly-up position because, deprived of visual cues, it can rely on the direction of the gravitational response centrifugal field only to distinguish 'up' from 'down'.

\section{Results}

At one $g$, before each maneuver, the fish swam at random with no observable behavioral abnormalities. The unavoidable circumstances of engine noise, vibration, possible oxygen shortage, or slight water temperature changes did not greatly influence the fish's spontaneous behavior. Provided the plane flew smoothly, sharp turns of the fish in any direction were rarely observed, and they were usually horizontally oriented. The behavioral responses of the fish to changes in the $g$ load are illustrated in Figure 2. Plottings of the data obtained by evaluation of single frames of the recording camera are shown in Figures 3 and 4.

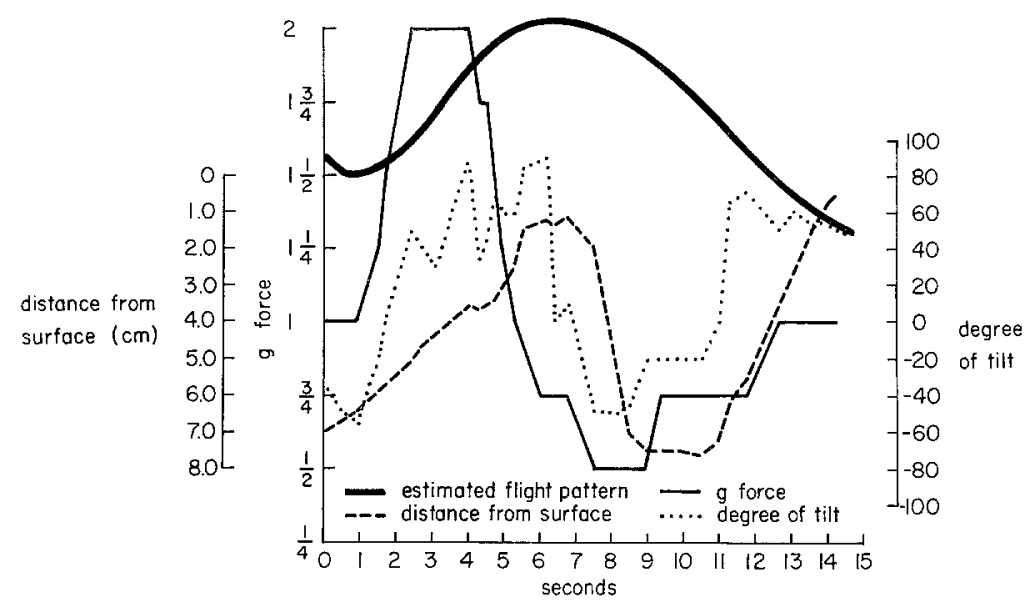

Fig. 3. Tilt and position of a goldfish during increasing $g$ load, followed by decreasing $g$ load during a parabolic flight. The values of $g$, tilt and position (distance from surface) in this and Figure 4 were obtained from consecutive single frames of a movie. Note the climb of the fish with increasing $g$ and the dive with decreasing $g$ and the latency between tilt and position.

(A) Transition Towards Lower ' $g$ ' (Figure 2a): When the $g$ force was lowered during a parabolic flight pattern, all fish reacted within $0.5-1.5 \mathrm{sec}$ of the $g$ meter deflection by tilting their longitudinal axis downward and propelling themselves forward.

The net result was a downward displacement. Often the magnitude of this displacement was limited by the bottom of the tank, for once reaching this barrier, a fish would persist in pressing its nose against it as if attempting to penetrate it. 


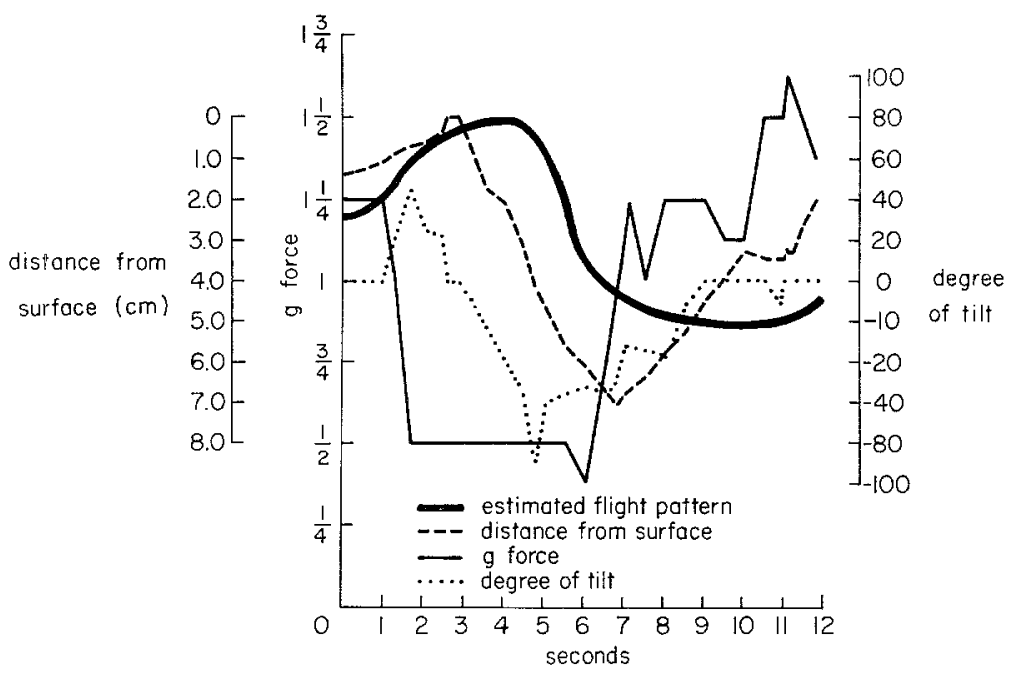

Fig. 4. Tilt and position of another goldfish during a parabolic flight, followed by a high load. These plottings confirm the experiment of Figure 3 and show that the sequence of decreasing and increasing $g$ is not critical for the effect.

When the gravitational forces returned to the original value, the longitudinal axis again assumed a horizontal or upwards pointing position, and the downward movement ceased. The latency of the downward movement was always longer than that of the tilting response. This downward movement was even further delayed if prior to the reduction in the $g$ force, the fish had been slightly pointed upward, for then the fish had first to rotate its longitudinal axis through the horizontal position before it could begin the downward movement.

(B) Transition Towards Higher ' $g$ ' (Figure 2b): When greater than one $g$ trajectories were flown, the fish consistently reacted by tilting upward and swimming forward so that an elevated position was reached. Occasionally, this movement appeared to be limited by the surface of the water. The latency for tilting and position change was of a magnitude similar to that in the low $g$ maneuvers, except for the fact that under conditions of increasing $g$ reactions were always less rapid and less pronounced.

(C) Combined Effects: Since for technical reasons the weightlessness parabola was entered in most cases from a higher than one $g$ load, and similarly since, some high $g$ maneuvers were started at the end of a weightless parabola when the plane was already in a diving position, combined effects were commonly observed.

When switching from an increasing to a decreasing $g$ load, the fish promptly changed from a slow upward movement to a quick downward movement in the tank. The latter movement was preceded by rotation of its longitudinal axis from the upward pointing position all the way to the downward pointing position. The film analysis indicates that the fish accomplished this rotation by a forward swimming movement, fanning its fins to initiate the direction change which indeed is the prevalent way fish achieve vertical course changes. This forward movement caused the fish first to rise 
a short distance in the tank before it entered the dive, the trajectory resembling a man's jump from a diving board.

Reciprocal movements were observed when at the end of a weightlessness parabola, lower than one $g$ forces gave way to higher than one $g$ forces.

(D) No Effect of Altitude Change: When a variometer indicating altitude change was observed together with the fish in the tank, it was noted that the fish did not react in any measurable way to altitude changes that were unaccompanied by vertical accelerations.

(E) Effect of Visual Cues: The reactions to increasing and decreasing $g$ forces could be obtained on a reliable basis only when the fish were blinded before the experiment. Experiments in which a blind and normal fish were placed in the same tank revealed that only the former displayed the described response to changes in gravitational forces. This experiment confirms the finding that if sight is intact, visual cues can override vestibular experiences (VON HoLST, 1950).

(F) Effect of Labyrinthectomy: A blind labyrinthectomized goldfish did not display the mentioned responses to decreasing or increasing $g$ whereas blind control fish with intact labyrinths flown at the same time in the same tank showed the response.

(G) Statistical Analysis: For each experiment, comparisons were made of the mean values of the fish's tilt and position during the last $3 \mathrm{sec}$ of control before the low or high $g$ maneuver was performed, with corresponding values obtained under

TABLE I

Statistical analysis

\begin{tabular}{cccc}
\hline & Difference of & \\
Number of & Mean Control and & Significance \\
Lean & Trials & Mean Experimental & Values \\
\hline
\end{tabular}

Direction of $g$ change: decreasing

Degree of Downward Tilt Position Change downwards $g$ change (decreasing)

$\begin{array}{ll}11 & 42.7^{\circ} \pm 11.0^{\circ} \\ 11 & 3.3 \pm 0.5 \mathrm{~cm} \\ 11 & 0.86 \pm 0.08 \mathrm{~g}\end{array}$

Direction of $g$ change: increasing

Degree of Downward Tilt Position Change upwards $g$ change (increasing)

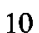

10

10

$$
\begin{aligned}
& 29.3^{\circ} \pm 11.7^{\circ} \\
& 2.2 \pm 0.8 \mathrm{~cm} \\
& 0.77 \pm 0.05 \mathrm{~g}
\end{aligned}
$$

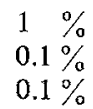

$0.1 \%$

$\begin{array}{lcr}0 & 29.3^{\circ} \pm 11.7^{\circ} & 5 \% \\ 0 & 2.2 \pm 0.8 \mathrm{~cm} & 5 \% \\ 0 & 0.77 \pm 0.05 \mathrm{~g} & 0.1 \%\end{array}$

conditions of maximum change in the $g$ load achieved during the course of the experiment (Table I). There were 11 such cases going from high to low $g$, and 10, from low to high; no data were eliminated. The difference between the mean values recorded under control and the peak or minimal $g$-load during the experiment was calculated and tested for statistical significance: the tilt change during decreasing $g$ reached the 
significance level of 0.01 and the change during increasing $g 0.05$. The fish's position change during decreasing $g$ was highly significant at the 0.001 level, and during increasing $g$ at a 0.05 level.

\section{Discussion}

The reactions of goldfish to increasing and decreasing $g$ forces pose a question as to whether only the changing $g$ load is responsible, or whether other factors play a role. The tank was airtight so changes in atmospheric pressure can be eliminated as a factor. Also, the possibility that changes in altitude per se can be sensed is unlikely, because altitude variations as indicated by a variometer on the tank, did not cause any measurable effects on the fish when unaccompanied by acceleration or deceleration. We, therefore, conclude that only changes in the $g$ load are responsible for the observed behavior.

The question arises as to which organs of the fish function as receptors for the stimuli evoking the observed reactions. At approaching weightlessness, the weights of all objects including the fish decrease and equalize to zero. As a consequence of decreasing external water pressure at this time, the swimming bladder expands, and at least theoretically, it is possible that the fish might try to compensate for this by diving down (such maneuvers would help him only in a normal gravitational field). However, our records indicate that although at every height in the fish tank, the swimming bladder is subjected to different pressures and at the very surface to a negligible pressure, the fish reactions were identical. The diving reaction to lowered $g$ force was even observable, when the enclosed fish tank was filled up to the top with water and did not allow the swimming bladder to change markedly its volume. This observation suggests that the swimming bladder is not involved in the reflex under consideration.

The intestines, which are suspended by ligaments to the body wall cannot be excluded on the basis of our experiments as being receptor areas for a response to decreasing $g$. However, since it is well known, that the vestibular organ contains special gravity receptors, it appears most likely that the principal receptors are in the otolith-macula system. After labyrinthectomy the response did not appear any more in the present study.

As far as the general theoretical background is concerned, there is no consensus among researchers in the field regarding which direction of otolith displacement constitutes the adequate stimulus for the otolith reflexes (LOWENSTEIN and ROBERTS, 1950; Magnus, 1924; Von HoLST, 1950). - Newer investigations (Ades and ENGSTROEM, 1965; LOWENSTEIN, 1966; WeRSÄLL and LUNDQUIST, 1966) indicate that the otolith system can sense different directions of displacement.

Regardless of the controversial opinions about the adequate or most effective direction of otolith-displacement, the question can be posed how the central nervous system processes these impulses; in other words, what kinds of reflexes eventually result from different directions of otolith displacement.

From a theoretical standpoint at least two opposing types of reflex behavior are 
possible. Otolith displacement could lead to movements of the whole organism into the direction of such displacements, or $180^{\circ}$ against such displacements. The former case would represent an ideal inertial stabilization system in space because it would sense passive displacement of the organism and counteract them by active movements which are aimed to restore any original position in space; the latter would be an ideal system for stabilization within a gravitational field, since it is able to restore the original direction of this field by active movements which reflectorily follow any changes of the direction in which gravity pulls. Unfortunately, neither reflex could subserve both purposes. Unless the nervous system can by its plasticity (learning) readjust itself to such different environmental conditions as weightlessness and gravity, or the remote possibility that it switches its reflex pattern 'at will' from gravitational to inertial stabilization, it appears not possible to handle meaningfully the influence of gravity and inertia on the otolith-system by the same organism. The responses of animals to otolith displacements imposed by tilt of the whole body (MAGNus, 1924; Von Holst, 1950) and to linear accelerations (HaRden Jones, 1956), lead us to conclude that animals have a behavioral tendency to correct all displacements of the otoliths by counterdirectional movements of the body, the correction being aimed $180^{\circ}$ against the direction of the otolith displacement. That is, the system works as a gravitational stabilizer. The experiment reported in this paper further supports this view. In our case we did not change the position of the animal, but simply experimentally decreased the $g$ load on the otoliths. The fact that reversible reflexes resulted from such changes suggests that the otoliths were displaced from their normal position by elastic forces and returned to it after $1 \mathrm{~g}$ was reached again. The fish reacted to an upward displacement of their otoliths, as expected, with a downward movement of their body and vice versa. The fact, that this stereotyped behavior occurred regardless of the starting position of the fish's longitudinal axis indicates that the otolith receptor system in fish is three-dimensional, i.e. the system reacts not only to the amplitude but also to the direction of otolith displacement. This seems to be in good agreement with the views of LowENSTEIN (1966), ADES and ENGSTROEM (1965) and WERSÄLL and LUNDQUIST (1966), concerning a possible functional organization of macula receptors. We do not know why the reactions of fish to increased $g$ force are less marked than to decreasing $g$ force. A possible explanation is that the ventral displacement of the otoliths during increasing $g$ is anatomically more restricted by the supporting surface than a dorsal displacement during decreasing $g$.

From an evolutionary standpoint it is understandable that the otolith reflexes were developed to help counteract gravity. The same system proves to be useful for stabilization of any preferred position within the field of gravity for all animals including fish. A slow tilting movement causes necessarily a larger displacement of an otolith by the change of direction in which gravity pulls than by enertia alone and only very rapid movements could reach the one $g$-level of acceleration through inertial forces. Passive forward movements of the fish induced by a water current would displace the otoliths backwards, and this displacement would result in an avalanche like accelerated movement forward of his body. The same would be true for passive 
backward and sideward movements with the result that instability would prevail. Obviously this is not the case so we must assume that at the ground the threshold of the system is too high for inertial reactions (BARLOW, 1964) or that the nervous system can suppress, when desirable, the reflex behavior. It appears, therefore, that the otolith system was developed to serve only one purpose, namely, to maintain proper attitude in the gravitational field. In space its role would hinder rather than support any useful orientation.

We do not know to what extent vestibular responses in animals apply to men. JONGKEES and GROEN (1946 and 1967) describe headdown tilting sensations of human subjects when the reciprocating movements of a parallel swing caused a forward displacement of the otoliths by inertia. CLARK (1967) reports that points in the visual field tend to move downwards during lowered $g$ and several persons reported an upsidedown 'illusion' during such a parabolic flight. In GraYBIEL and KeLLoG's (1966) experiments, two persons had to assume an inverted headdown position during parabolic flight and described their sensation in this inverted position as 'feetdown' and normal. Both observations seem to be paralleled to some degree by the behavior of our goldfish. It seems possible that an astronaut will have to learn to disregard such meaningless subjective sensations created by his transition from high $g$ to the weightless state, and also to overcome the tendency to react maladaptively albeit reflexively to displacement of his otoliths by any accidental inertial or centrifugal forces resulting from sudden changes in the position of his body or spacecraft during the state of weightlessness.

To date the number of our experiments is not sufficient to gain insight into other relevant questions, for instance, whether there are differences in the results when the experiments are conducted in the range between $1 \mathrm{~g}$ and $0 \mathrm{~g}$ compared to between $2 \mathrm{~g}$ and $1 \mathrm{~g}$. Furthermore, since our data indicate that the vestibular system of fish responds to increasing and decreasing $g$ forces (delta $g$ ), the question arises concerning how much and how fast adaptation occurs to this stimulus. Investigation of this adaptation problem requires weightlessness flights of longer duration than were possible to achieve in light aircrafts.

\section{Acknowledgment}

This work was supported by NASA Grant NGR 23-005-201.

\section{References}

Ades, H. W. and Engstroem, H.: 1965, in The Role of the Vestibular Organs in the Exploration of Space. NASA Publication SP-77. U.S. Naval School of Aviation Medicine, Pensacola, Fla., National Aeronautics and Space Administration, Washington, D.C., p. 23.

BARLOW, J. S.: 1964, J. Theoret. Biol. 6, 76.

Breuer, J.: 1891, Pfluegers Arch. Ges. Physiol. 48, 195.

ClaRK, B.: 1967, in Third Symposium on the Role of the Vestibular Organs in Space Exploration. NASA Publication SP-152. Naval Aerospace Medical Institute Naval Aerospace Medical Center, Pensacola, Fla., National Aeronautics and Space Administration, Washington, D.C., p. 331.

Gerathewohl, S. J., Ritter, O. L., and Stallings, H. D.: 1957, SAM Report \# 57. 
GraYBiel, A. and Kellog, R. S.: 1966, in Second Symposium on the Role of the Vestibular Organs in Space Exploration. NASA Publication SP-115. AMES Research Center, Moffett Field, California. National Aeronautics and Space Administration, Washington, D.C., p. 15.

HABER, F. and HABER, H.: 1950, The Journal of Aviation Medicine 21, 395.

HARDEN JONES, F. R.: 1956, Nature 178, 642.

Hawkins, W. R.: 1960, in Lectures in Aerospace Medicine. School of Aviation Medicine, ch. 11 'Space flight dynamics II, Zero G', p. 11.

Howard, I. P. and Templeton, W. B.: 1966, in Human Spatial Orientation. John Wiley and Sons, London, ch. 16,5, pp. 436 and 438.

JongkeEs, L. B. W.: 1967, in Third Symposium on the Role of the Vestibular Organs in Space Exploration. NASA Publication SP-152. Naval Aerospace Medical Institute Naval Aerospace Medical Center, Pensacola, Fla., National Aeronautics and Space Administration, Washington, D.C., p. 307.

JongkeEs, L. B. W. and Groen, J. J.: 1946, J. Laryngol. Otol. 61, 529.

LowensteIn, O.: 1966, in Second Symposium on the Role of the Vestibular Organs in Space Exploration. NASA Publication SP-115. AMES Research Center, Moffett Field, California, 1966. National Aeronautics and Space Administration, Washington, D.C. p. 73.

Lowenstein, O. and Roberts, T. M. D.: 1950, J. Physiol. 110, 392.

MaGnus, R.: 1924, Körperstellung, Springer, London, New York, Berlin.

Von Holst, E.: 1950, Z. Vergleich. Physiol. 32, 60.

WARD, J. E.: 1957, SAM Report \# 57-121.

Wersäld, J. and LundQVIST, P. G.: 1966, in Second Symposium on the Role of the Vestibular Organs in Space Exploration. NASA Publication SP-115. AMES Research Center Moffett Field, California. National Aeronautics and Space Administration, Washington, D.C., p. 57. 